\title{
A Study of Multiple Integrals with Maple
}

\author{
Chii-Huei Yu \\ Department of Management and Information, Nan Jeon University of Science and Technology, Tainan City, 73746, Taiwan \\ *Corresponding Author: chiihuei@mail.njtc.edu.tw
}

Copyright (C) 2014 Horizon Research Publishing All rights reserved.

\begin{abstract}
The multiple integral problem is closely related with probability theory and quantum field theory. This paper uses the mathematical software Maple for the auxiliary tool to study two types of multiple integrals. We can obtain the infinite series forms of these two types of multiple integrals by using integration term by term theorem. In addition, we provide some examples to do calculation practically. The research methods adopted in this study involved finding solutions through manual calculations and verifying these solutions by using Maple. This type of research method not only allows the discovery of calculation errors, but also helps modify the original directions of thinking from manual and Maple calculations. For this reason, Maple provides insights and guidance regarding problem-solving methods.
\end{abstract}

Keywords Multiple Integrals, Infinite Series Forms, Integration Term By Term Theorem, Maple

\section{Introduction}

The computer algebra system (CAS) has been widely employed in mathematical and scientific studies. The rapid computations and the visually appealing graphical interface of the program render creative research possible. Maple possesses significance among mathematical calculation systems and can be considered a leading tool in the CAS field. The superiority of Maple lies in its simple instructions and ease of use, which enable beginners to learn the operating techniques in a short period. In addition, through the numerical and symbolic computations performed by Maple, the logic of thinking can be converted into a series of instructions. The computation results of Maple can be used to modify our previous thinking directions, thereby forming direct and constructive feedback that can aid in improving understanding of problems and cultivating research interests. Inquiring through an online support system provided by Maple or browsing the Maple website (www.maplesoft.com) can facilitate further understanding of Maple and might provide unexpected insights. As for the instructions and operations of Maple, we can refer to [1-7].

The multiple integral problem is closely related with probability theory and quantum field theory, and can refer to [8-9]. For this reason, the evaluation and numerical calculation of multiple integrals is important. In this study, we evaluate the following two types of ${ }^{n}$-tuple integrals

$$
\begin{gathered}
\int_{0}^{1} \cdots \int_{0}^{1} f\left(\prod_{m=1}^{n} x_{m}{ }^{r_{m}}\right) d x_{1} \cdots d x_{n} \\
\int_{1}^{\infty} \cdots \int_{1}^{\infty} g\left(\prod_{m=1}^{n} x_{m}{ }^{s_{m}}\right) d x_{1} \cdots d x_{n}
\end{gathered}
$$

Where $n$ is any positive integer, $r_{m}, s_{m}$ are real numbers, $r_{m} \geq 0, s_{m}>1$ for $m=1, . ., n$. And

$$
\begin{gathered}
f(t)=\frac{A_{1}}{t+a_{1}}+\cdots+\frac{A_{p}}{t+a_{p}} \\
g(t)=\frac{B_{1}}{t+b_{1}}+\cdots+\frac{B_{p}}{t+b_{p}}
\end{gathered}
$$

Where $p$ is a positive integer, $A_{j}, B_{j}, a_{j}, b_{j}$ are real numbers, and $\left|a_{j}\right|>1,\left|b_{j}\right|<1$ for all $j=1, . ., p$. We can obtain the infinite series forms of these two types of multiple integrals by using integration term by term theorem ; these are the major results of this study (i.e., Theorems 1 and 2). In addition, we obtain two corollaries from these two theorems. For the study of related multiple integral problems can refer to [10-23]. On the other hand, we propose some multiple integrals to do calculation practically. The research methods adopted in this study involved finding solutions through manual calculations and verifying these solutions by using Maple. This type of research method not only allows the discovery of calculation errors, but also helps modify the original directions of thinking from manual and Maple calculations. Therefore, Maple provides insights and guidance regarding problem-solving methods.

\section{Main Results}

Firstly, we introduce a notation and a formula used in this paper.

\subsection{Notation}


$\prod_{m=1}^{n} c_{m}=c_{1} \times c_{2} \times \cdots \times c_{n}$, where $n$ is a positive integer, $c_{m}$ are real numbers for $m=1, . ., n$.

\subsection{Geometric Series}

$\frac{1}{1+\beta}=\sum_{k=0}^{\infty}(-1)^{k} \beta^{k}$, where $\beta$ is a real number, $|\beta|<1$.

Next, we introduce an important theorem used in this study.

\subsection{Integration Term by Term Theorem ([24]).}

Suppose $\left\{g_{n}\right\}_{n=0}^{\infty}$ is a sequence of Lebesgue integrable functions defined on an interval $I$. If $\sum_{n=0}^{\infty} \int_{I}\left|g_{n}\right|$ is convergent, then $\int_{I} \sum_{n=0}^{\infty} g_{n}=\sum_{n=0}^{\infty} \int_{I} g_{n}$.

The following is the first major result in this study, we determine the infinite series form of the multiple integral (1).

\subsection{Theorem 1}

If $n$ is a positive integer, $r_{m}$ are real numbers, $r_{m} \geq 0$ for all $m=1, . ., n$. Suppose $p$ is a positive integer, $A_{j}, a_{j}$ are real numbers, and $\left|a_{j}\right|>1$ for all $j=1, . ., p$. Let the domain of the function

$$
f(t)=\frac{A_{1}}{t+a_{1}}+\cdots+\frac{A_{p}}{t+a_{p}}
$$

be $\{t \in R|| t \mid<1\}$. Then the $n$-tuple integral

$$
\begin{gathered}
\int_{0}^{1} \cdots \int_{0}^{1} f\left(\prod_{m=1}^{n} x_{m}^{r_{m}}\right) d x_{1} \cdots d x_{n} \\
=\sum_{k=0}^{\infty} \frac{(-1)^{k}}{\prod_{m=1}^{n}\left(r_{m} k+1\right)}\left(\sum_{j=1}^{p} \frac{A_{j}}{a_{j}{ }^{k+1}}\right)
\end{gathered}
$$

\subsubsection{Proof}

Because

$$
\begin{array}{r}
f(t)=\frac{A_{1}}{t+a_{1}}+\cdots+\frac{A_{p}}{t+a_{p}} \\
=\frac{A_{1}}{a_{1}} \cdot \frac{1}{1+\frac{t}{a_{1}}}+\cdots+\frac{A_{p}}{a_{p}} \cdot \frac{1}{1+\frac{t}{a_{p}}}
\end{array}
$$

$=\frac{A_{1}}{a_{1}} \cdot \sum_{k=0}^{\infty}(-1)^{k}\left(\frac{t}{a_{1}}\right)^{k}+\cdots+\frac{A_{p}}{a_{p}} \cdot \sum_{k=0}^{\infty}(-1)^{k}\left(\frac{t}{a_{p}}\right)^{k}$

(By geometric series because $|t|<1$ and $\left|a_{j}\right|>1$ for all $j=1, . ., p$ )

$$
=\sum_{k=0}^{\infty}(-1)^{k}\left(\sum_{j=1}^{p} \frac{A_{j}}{a_{j}^{k+1}}\right) t^{k}
$$

Therefore,

$$
\begin{gathered}
\int_{0}^{1} \cdots \int_{0}^{1} f\left(\prod_{m=1}^{n} x_{m}{ }^{r_{m}}\right) d x_{1} \cdots d x_{n} \\
=\lim _{\substack{\delta_{m} \rightarrow 1^{-} \\
1 \leq m \leq n}} \int_{0}^{\delta_{n}} \cdots \int_{0}^{\delta_{1}} f\left(\prod_{m=1}^{n} x_{m}{ }^{r_{m}}\right) d x_{1} \cdots d x_{n} \\
=\lim _{\substack{\delta_{m \rightarrow 1} \rightarrow 1^{-} \\
1 \leq m \leq n}} \int_{0}^{\delta_{n}} \cdots \int_{0}^{\delta_{1}} \sum_{k=0}^{\infty}(-1)^{k}\left(\sum_{j=1}^{p} \frac{A_{j}}{a_{j}{ }^{k+1}}\right)\left(\prod_{m=1}^{n} x_{m}{ }^{r_{m}}\right)^{k} d x_{1} \cdots d x_{n}
\end{gathered}
$$

(Using (6))

$=\lim _{\substack{\delta_{m \rightarrow 1^{-}} \\ 1 \leq m \leq n}} \sum_{k=0}^{\infty}(-1)^{k}\left(\sum_{j=1}^{p} \frac{A_{j}}{a_{j}{ }^{k+1}}\right) \int_{0}^{\delta_{n}} \cdots \int_{0}^{\delta_{1}} \prod_{m=1}^{n} x_{m}{ }^{{ }^{r} k} d x_{1} \cdots d x_{n}$

( By integration term by term theorem)

$$
\begin{gathered}
=\lim _{\substack{\delta_{m} \rightarrow 1^{-} \\
1 \leq m \leq n}} \sum_{k=0}^{\infty}(-1)^{k}\left(\sum_{j=1}^{p} \frac{A_{j}}{a_{j}{ }^{k+1}}\right) \prod_{m=1}^{n} \int_{0}^{\delta_{m}} x_{m}{ }^{r_{m} k} d x_{m} \\
=\lim _{\substack{\delta_{m} \rightarrow 1^{-} \\
1 \leq m \leq n}} \sum_{k=0}^{\infty}(-1)^{k}\left(\sum_{j=1}^{p} \frac{A_{j}}{a_{j}^{k+1}}\right) \prod_{m=1}^{n} \frac{\delta_{m}{ }^{r_{m} k+1}}{r_{m} k+1} \\
=\sum_{k=0}^{\infty} \frac{(-1)^{k}}{\prod_{m=1}^{n}\left(r_{m} k+1\right)}\left(\sum_{j=1}^{p} \frac{A_{j}}{a_{j}^{k+1}}\right)
\end{gathered}
$$

In Theorem 1, taking $x_{m}=e^{-w_{m}}$ for all $m=1, . ., n$, we immediately have the following result.

\subsection{Corollary 1}

If the assumptions are the same as Theorem 1, then the $n$-tuple improper integral

$$
\begin{gathered}
\int_{0}^{\infty} \cdots \int_{0}^{\infty} \exp \left(-\sum_{m=1}^{n} w_{m}\right) f\left(\exp \left(-\sum_{m=1}^{n} r_{m} w_{m}\right)\right) d w_{1} \cdots d w_{n} \\
=\sum_{k=0}^{\infty} \frac{(-1)^{k}}{\prod_{m=1}^{n}\left(r_{m} k+1\right)}\left(\sum_{j=1}^{p} \frac{A_{j}}{a_{j}^{k+1}}\right)
\end{gathered}
$$


The following is the second major result in this paper, we obtain the infinite series forms of the multiple integral (2).

\subsection{Theorem 2}

If $n$ is a positive integer, $s_{m}$ are real numbers, $s_{m}>1$ for all $m=1, \ldots, n$. Suppose $p$ is a positive integer, $B_{j}, b_{j}$ are real numbers, and $\left|b_{j}\right|<1$ for all $j=1, . ., p$. Suppose the domain of the function

$$
g(t)=\frac{B_{1}}{t+b_{1}}+\cdots+\frac{B_{p}}{t+b_{p}}
$$

is $\{t \in R|| t \mid>1\}$. Then the $n$-tuple improper integral

$$
\begin{aligned}
& \int_{1}^{\infty} \cdots \int_{1}^{\infty} g\left(\prod_{m=1}^{n} x_{m}{ }^{s_{m}}\right) d x_{1} \cdots d x_{n} \\
= & \sum_{k=0}^{\infty} \frac{(-1)^{k}}{\prod_{m=1}^{n}\left(s_{m} k+s_{m}-1\right)}\left(\sum_{j=1}^{p} B_{j} b_{j}{ }^{k}\right)
\end{aligned}
$$

\subsubsection{Proof}

Because

$$
\begin{gathered}
g(t)=\frac{B_{1}}{t+b_{1}}+\cdots+\frac{B_{p}}{t+b_{p}} \\
=\frac{B_{1}}{t} \cdot \frac{1}{1+\frac{b_{1}}{t}}+\cdots+\frac{B_{p}}{t} \cdot \frac{1}{1+\frac{b_{p}}{t}} \\
=\frac{B_{1}}{t} \cdot \sum_{k=0}^{\infty}(-1)^{k}\left(\frac{b_{1}}{t}\right)^{k}+\cdots+\frac{B_{p}}{t} \cdot \sum_{k=0}^{\infty}(-1)^{k}\left(\frac{b_{p}}{t}\right)^{k}
\end{gathered}
$$

(Using geometric series because $|t|>1$ and $\left|b_{j}\right|<1$ for all $j=1, . ., p)$

$$
=\sum_{k=0}^{\infty}(-1)^{k}\left(\sum_{j=1}^{p} B_{j} b_{j}^{k}\right) t^{-k-1}
$$

Thus,

$$
\begin{gathered}
\int_{1}^{\infty} \cdots \int_{1}^{\infty} g\left(\prod_{m=1}^{n} x_{m}{ }^{s_{m}}\right) d x_{1} \cdots d x_{n} \\
=\lim _{\substack{\lambda_{m} \rightarrow 1^{+} \\
1 \leq m \leq n}} \int_{\lambda_{n}}^{\infty} \cdots \int_{\lambda_{1}}^{\infty} g\left(\prod_{m=1}^{n} x_{m}{ }^{s_{m}}\right) d x_{1} \cdots d x_{n} \\
=\lim _{\substack{\lambda_{m} \rightarrow 1^{+} \\
1 \leq m \leq n}} \int_{\lambda_{n}}^{\infty} \cdots \int_{\lambda_{1}}^{\infty} \sum_{k=0}^{\infty}(-1)^{k}\left(\sum_{j=1}^{p} B_{j} b_{j}{ }^{k}\right)\left(\prod_{m=1}^{n} x_{m}{ }^{s_{m}}\right)^{-k-1} d x_{1} \cdots d x_{n}
\end{gathered}
$$

(By (9))

$$
=\lim _{\substack{\lambda_{m \rightarrow 1^{+}} \rightarrow 1^{1 \leq m \leq n} \\ k=0}} \sum^{\infty}(-1)^{k}\left(\sum_{j=1}^{p} B_{j} b_{j}{ }^{k}\right) \int_{\lambda_{n}}^{\infty} \cdots \int_{\lambda_{1}}^{\infty} \prod_{m=1}^{n} x_{m}{ }^{-s_{m} k-s_{m}} d x_{1} \cdots d x_{n}
$$

( Using integration term by term theorem)

$$
\begin{gathered}
=\lim _{\substack{\lambda_{m} \rightarrow 1^{+} \\
1 \leq m \leq n}} \sum_{k=0}^{\infty}(-1)^{k}\left(\sum_{j=1}^{p} B_{j} b_{j}{ }^{k}\right) \prod_{m=1}^{n} \int_{\lambda_{m}}^{\infty} x_{m}{ }^{-s_{m} k-s_{m}} d x_{m} \\
=\lim _{\substack{\lambda_{m} \rightarrow 1^{+} \\
1 \leq m \leq n}} \sum_{k=0}^{\infty}(-1)^{k}\left(\sum_{j=1}^{p} B_{j} b_{j}{ }^{k}\right) \prod_{m=1}^{n} \frac{-\lambda_{m}{ }^{-s_{m} k-s_{m}+1}}{-s_{m} k-s_{m}+1} \\
=\sum_{k=0}^{\infty} \frac{(-1)^{k}}{\prod_{m=1}^{n}\left(s_{m} k+s_{m}-1\right)}\left(\sum_{j=1}^{p} B_{j} b_{j}^{k}\right)
\end{gathered}
$$

In Theorem 2, let $x_{m}=e^{u_{m}}$ for all $m=1, . ., n$, then the following result holds.

\subsection{Corollary 2}

If the assumptions are the same as Theorem 2, then the $n$ -tuple improper integral

$$
\begin{gathered}
\int_{0}^{\infty} \cdots \int_{0}^{\infty} \exp \left(\sum_{m=1}^{n} u_{m}\right) g\left(\exp \left(\sum_{m=1}^{n} s_{m} u_{m}\right)\right) d u_{1} \cdots d u_{n} \\
=\sum_{k=0}^{\infty} \frac{(-1)^{k}}{\prod_{m=1}^{n}\left(s_{m} k+s_{m}-1\right)}\left(\sum_{j=1}^{p} B_{j} b_{j}{ }^{k}\right)
\end{gathered}
$$

\section{Examples}

In the following, for the two types of multiple integrals in this study, we provide some examples and use Theorems 1, 2 and Corollaries 1, 2 to determine the infinite series forms of these multiple integrals. On the other hand, we employ Maple to calculate the approximations of these multiple integrals and their solutions for verifying our answers.

\subsection{Example 1}

Let the domain of the function

$$
f_{1}(t)=\frac{t^{2}+33 t-118}{t^{3}-5 t^{2}-2 t+24}
$$

be $\{t \in R|| t \mid<1\}$, and let $r_{1}, r_{2}, r_{3} \geq 0$. Because

$$
f_{1}(t)=\frac{5}{t-4}+\frac{-6}{t+2}+\frac{2}{t-3}
$$


(The case of $A_{1}=5, A_{2}=-6, A_{3}=2, a_{1}=-4, a_{2}=2, a_{3}=-3$ in Theorem 1). Thus, by Theorem 1, we obtain the triple integral

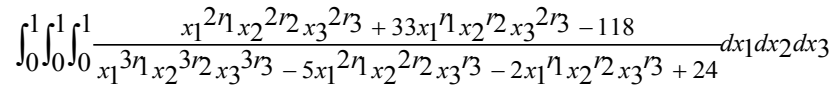

$$
\begin{aligned}
& =\sum_{k=0}^{\infty} \frac{(-1)^{k}}{\left(r_{1} k+1\right)\left(r_{2} k+1\right)\left(r_{3} k+1\right)}\left(\frac{5}{(-4)^{k+1}}-\frac{6}{2^{k+1}}+\frac{2}{(-3)^{k+1}}\right)
\end{aligned}
$$

Hence, we obtain

$$
\begin{aligned}
& \int_{0}^{1} \int_{0}^{1} \int_{0}^{1} \frac{x_{1}{ }^{2} x_{2}{ }^{4} x_{3}{ }^{6}+33 x_{1} x_{2}{ }^{2} x_{3}{ }^{3}-118}{x_{1}{ }^{3} x_{2}{ }^{6} x_{3}{ }^{9}-5 x_{1}{ }^{2} x_{2}{ }^{4} x_{3}{ }^{6}-2 x_{1} x_{2}{ }^{2} x_{3}{ }^{3}+24} d x_{1} d x_{2} d x_{3} \\
& =\sum_{k=0}^{\infty} \frac{(-1)^{k}}{(k+1)(2 k+1)(3 k+1)}\left(\frac{5}{(-4)^{k+1}}-\frac{6}{2^{k+1}}+\frac{2}{(-3)^{k+1}}\right)
\end{aligned}
$$

In the following, we use Maple to verify the correctness of (14).

$>$ evalf(Tripleint $\left(\left(\mathrm{x} 1^{\wedge} 2 * \mathrm{x} 2^{\wedge} 4 * \mathrm{x} 3^{\wedge} 6+33^{*} \mathrm{x} 1^{*} \mathrm{x} 2^{\wedge} 2 * \mathrm{x} 3^{\wedge} 3-118\right)\right.$ $/\left(\mathrm{x} 1^{\wedge} 3^{*} \mathrm{x} 2^{\wedge} 6^{*} \mathrm{x} 3^{\wedge} 9-5^{*} \mathrm{x} 1^{\wedge} 2^{*} \mathrm{x} 2^{\wedge} 4^{*} \mathrm{x} 3^{\wedge} 6-2^{*} \mathrm{x} 1^{*} \mathrm{x} 2^{\wedge} 2^{*} \mathrm{x} 3^{\wedge} 3+2\right.$ $4), \mathrm{x} 1=0 . .1, \mathrm{x} 2=0 . .1, \mathrm{x} 3=0 . .1), 14)$;

$-4.8841370709055$

$>$ evalf $\left(\operatorname{sum}\left((-1)^{\wedge} \mathrm{k} /((\mathrm{k}+1) *(2 * \mathrm{k}+1) *(3 * \mathrm{k}+1)) *\left(5 /(-4)^{\wedge}(\mathrm{k}+1)-\right.\right.\right.$ $\left.6 / 2^{\wedge}(\mathrm{k}+1)+2 /(-3)^{\wedge}(\mathrm{k}+1)\right), \mathrm{k}=0$...infinity $\left.), 14\right)$; $-4.8841370709060$

\subsection{Example 2}

Suppose the domain of the function

$$
f_{2}(t)=\frac{-2 t-22}{t^{2}-2 t-8}
$$

is $\{t \in R|| t \mid<1\}$, and let $r_{1}, r_{2} \geq 0$. Because

$$
f_{2}(t)=\frac{3}{t+2}+\frac{-5}{t-4}
$$

(The case of $A_{1}=3, A_{2}=-5, a_{1}=2, a_{2}=-4$ in Corollary 1). Therefore, by Corollary 1 , we have the double improper integral

$$
\begin{gathered}
\int_{0}^{\infty} \int_{0}^{\infty} \frac{\exp \left(-w_{1}-w_{2}\right)\left[-2 \exp \left(-r_{1} w_{1}-r_{2} w_{2}\right)-22\right]}{\exp \left(-2 r_{1} w_{1}-2 r_{2} w_{2}\right)-2 \exp \left(-r_{1} w_{1}-r_{2} w_{2}\right)-8} d w_{1} d w_{2} \\
=\sum_{k=0}^{\infty} \frac{(-1)^{k}}{\left(r_{1} k+1\right)\left(r_{2} k+1\right)}\left(\frac{3}{2^{k+1}}-\frac{5}{(-4)^{k+1}}\right)
\end{gathered}
$$

Hence, we can determine

$\int_{0}^{\infty} \int_{0}^{\infty} \frac{\exp \left(-w_{1}-w_{2}\right)\left[-2 \exp \left(-2 w_{1}-w_{2}\right)-22\right]}{\exp \left(-4 w_{1}-2 w_{2}\right)-2 \exp \left(-2 w_{1}-w_{2}\right)-8} d w_{1} d w_{2}$

$$
=\sum_{k=0}^{\infty} \frac{(-1)^{k}}{(2 k+1)(k+1)}\left(\frac{3}{2^{k+1}}-\frac{5}{(-4)^{k+1}}\right)
$$

We also use Maple to verify the correctness of (18).
$>$ evalf(Doubleint $(\exp (-\mathrm{w} 1-\mathrm{w} 2) *(-2 * \exp (-2 * \mathrm{w} 1-\mathrm{w} 2)-22) /$ $(\exp (-4 * \mathrm{w} 1-2 * \mathrm{w} 2)-2 * \exp (-2 * \mathrm{w} 1-\mathrm{w} 2)-8), \mathrm{w} 1=0$...infinity,w2 $=0$..infinity), 14$)$;

2.7029842891882

$>\operatorname{evalf}\left(\operatorname{sum}\left((-1)^{\wedge} \mathrm{k} /((2 * \mathrm{k}+1) *(\mathrm{k}+1))^{*}\left(3 / 2^{\wedge}(\mathrm{k}+1)-5 /(-4)^{\wedge}(\mathrm{k}+1\right.\right.\right.$ )$), \mathrm{k}=0$...infinity), 14$)$;

2.7029842891882

\subsection{Example 3}

Assume the domain of the function

$$
g_{1}(t)=\frac{29 t-12}{6 t^{2}-5 t+1}
$$

is $\{t \in R|| t \mid>1\}$, and let $s_{1}, s_{2}>1$. Because

$$
g_{1}(t)=\frac{5 / 2}{t-1 / 2}+\frac{7 / 3}{t-1 / 3}
$$

(The case of $B_{1}=5 / 2, B_{2}=7 / 3, b_{1}=-1 / 2, b_{2}=-1 / 3$ in Theorem 2). Thus, using Theorem 2, we obtain the double improper integral

$$
\begin{array}{r}
\int_{1}^{\infty} \int_{1}^{\infty} \frac{29 x_{1}{ }^{s_{1}} x_{2}{ }^{s_{2}}-12}{6 x_{1}{ }^{2 s_{1}} x_{2}{ }^{2 s_{2}}-5 x_{1}{ }^{s_{1}} x_{2}{ }^{s_{2}}+1} d x_{1} d x_{2} \\
=\sum_{k=0}^{\infty} \frac{(-1)^{k}}{\left(s_{1} k+s_{1}-1\right)\left(s_{2} k+s_{2}-1\right)}\left(\frac{5}{2}\left(-\frac{1}{2}\right)^{k}+\frac{7}{3}\left(-\frac{1}{3}\right)^{k}\right)
\end{array}
$$

Therefore, we have

$$
\begin{gathered}
\int_{1}^{\infty} \int_{1}^{\infty} \frac{29 x_{1}{ }^{4} x_{2}{ }^{3}-12}{6 x_{1}{ }^{8} x_{2}{ }^{6}-5 x_{1}{ }^{4} x_{2}{ }^{3}+1} d x_{1} d x_{2} \\
=\sum_{k=0}^{\infty} \frac{(-1)^{k}}{(4 k+3)(3 k+2)}\left(\frac{5}{2} \cdot\left(-\frac{1}{2}\right)^{k}+\frac{7}{3} \cdot\left(-\frac{1}{3}\right)^{k}\right)
\end{gathered}
$$

Using Maple to verify the correctness of (22) as follows: $>$ evalf(Doubleint $\left(\left(29^{*} \mathrm{x} 1^{\wedge} 4 * \mathrm{x} 2^{\wedge} 3-12\right) /\left(6^{*} \mathrm{x} 1^{\wedge} 8^{*} \mathrm{x} 2^{\wedge} 6-5^{*} \mathrm{x} 1^{\wedge}\right.\right.$ $4 * \times 2 \wedge 3+1), \times 1=1$..infinity, $\mathrm{x} 2=1$..infinity), 14$)$;

0.87700403798300

$>$ evalf $\left(\operatorname{sum}\left((-1)^{\wedge} \mathrm{k} /((4 * \mathrm{k}+3) *(3 * \mathrm{k}+2)) *\left(5 / 2 *(-1 / 2)^{\wedge} \mathrm{k}+7 / 3 *(-\right.\right.\right.$ $\left.1 / 3)^{\wedge} \mathrm{k}\right), \mathrm{k}=0$..infinity), 16);

\subsection{9}

\subsection{Example 4}

Let the domain of the function

$$
g_{2}(t)=\frac{19 t+1}{12 t^{2}+11 t+2}
$$

be $\{t \in R|| t \mid>1\}$, and let $s_{1}, s_{2}>1$. Because

$$
g_{2}(t)=\frac{7 / 3}{t+2 / 3}+\frac{-3 / 4}{t+1 / 4}
$$

(The case of $B_{1}=7 / 3, B_{2}=-3 / 4, b_{1}=2 / 3, b_{2}=1 / 4$ in Corollary 2 ). Hence, by Corollary 2 , we obtain the double 
improper integral

$$
\begin{aligned}
& \int_{0}^{\infty} \int_{0}^{\infty} \frac{\exp \left(u_{1}+u_{2}\right) \cdot\left[19 \exp \left(s_{1} u_{1}+s_{2} u_{2}\right)+1\right]}{12 \exp \left(2 s_{1} u_{1}+2 s_{2} u_{2}\right)+11 \exp \left(s_{1} u_{1}+s_{2} u_{2}\right)+2} d u_{1} d u_{2} \\
= & \sum_{k=0}^{\infty} \frac{(-1)^{k}}{\left(s_{1} k+s_{1}-1\right)\left(s_{2} k+s_{2}-1\right)}\left(\frac{7}{3} \cdot\left(\frac{2}{3}\right)^{k}-\frac{3}{4} \cdot\left(\frac{1}{4}\right)^{k}\right)(25)
\end{aligned}
$$

Thus, we can evaluate

$$
\begin{gathered}
\int_{0}^{\infty} \int_{0}^{\infty} \frac{\exp \left(u_{1}+u_{2}\right) \cdot\left[19 \exp \left(2 u_{1}+5 u_{2}\right)+1\right]}{12 \exp \left(4 u_{1}+10 u_{2}\right)+11 \exp \left(2 u_{1}+5 u_{2}\right)+2} d u_{1} d u_{2} \\
\quad=\sum_{k=0}^{\infty} \frac{(-1)^{k}}{(2 k+1)(5 k+4)}\left(\frac{7}{3} \cdot\left(\frac{2}{3}\right)^{k}-\frac{3}{4} \cdot\left(\frac{1}{4}\right)^{k}\right)
\end{gathered}
$$

Verifying the correctness of (26) as follows:

$>$ evalf $($ Doubleint $(\exp (\mathrm{u} 1+\mathrm{u} 2) *(19 * \exp (2 * \mathrm{u} 1+5 * \mathrm{u} 2)+1) /(12$

$* \exp (4 * \mathrm{u} 1+10 * \mathrm{u} 2)+11 * \exp (2 * \mathrm{u} 1+5 * \mathrm{u} 2)+2), \mathrm{u} 1=0$...infinity, $\mathrm{u} 2=0$..infinity), 14);

0.35566818033965

$>\operatorname{evalf}\left(\operatorname{sum}\left((-1)^{\wedge} \mathrm{k} /((2 * \mathrm{k}+1) *(5 * \mathrm{k}+4)) *\left(7 / 3 *(2 / 3)^{\wedge} \mathrm{k}-3 / 4 *(1 /\right.\right.\right.$

$\left.4)^{\wedge} \mathrm{k}\right), \mathrm{k}=0$...infinity), 14$)$;

0.35566818033959

\section{Conclusions}

As mentioned, the multiple integral problem is important in probability theory and quantum field theory. In this study, we propose a new technique to solve two types of multiple integrals, and we hope this method can be applied in mathematical statistics or quantum physics. Simultaneously, we know the integration term by term theorem plays a significant role in the theoretical inferences of this paper. In fact, the application of this theorem is extensive, and can be used to easily solve many difficult problems; we endeavor to conduct further studies on related applications. On the other hand, Maple also plays a vital assistive role in problem-solving. In the future, we will extend the research topic to other calculus and engineering mathematics problems and solve these problems by using Maple. These results will be used as teaching materials for Maple on education and research to enhance the connotations of calculus and engineering mathematics.

\section{REFERENCES}

[1] R. J. Stroeker and J. F. Kaashoek, Discovering Mathematics with Maple : An Interactive Exploration for Mathematicians, Engineers and Econometricians, Basel: Birkhauser Verlag, 1999.

[2] C. T. J. Dodson and E. A. Gonzalez, Experiments in Mathematics Using Maple, New York: Springer-Verlag, 1995.

[3] F. Garvan, The Maple Book, London: Chapman \& Hall/CRC,
2001.

[4] D. Richards, Advanced Mathematical Methods with Maple, New York: Cambridge University Press, 2002.

[5] J. S. Robertson, Engineering Mathematics with Maple, New York: McGraw-Hill, 1996.

[6] C. Tocci and S. G. Adams, Applied Maple for Engineers and Scientists, Boston: Artech House, 1996.

[7] M. L. Abell and J. P. Braselton, Maple by Example, 3rd ed., New York: Elsevier Academic Press, 2005.

[8] F. Streit, On multiple integral geometric integrals and their applications to probability theory, Canadian Journal of Mathematics, Vol. 22, pp. 151-163, 1970.

[9] L. H. Ryder, Quantum Field Theory, 2nd ed., New York: Cambridge University Press, 1996.

[10] C.-H. Yu, A study on the multiple improper integral problems with Maple, Applied Mechanics and Materials, in press.

[11] C. -H. Yu, Using Maple to study the multiple improper integral problem, Proceedings of IIE Asian Conference 2013, Taiwan, Vol. 1, pp. 625-632, 2013.

[12] C. - H. Yu, A study on the multiple improper integral problems, Journal of Hsin Sheng, in press.

[13] C. -H. Yu, Application of Maple: taking the evaluation of double improper integrals as an example, Proceedings of the 7th International Conference on Information Technology, Taiwan, No.28, 2013.

[14] C. -H. Yu, Application of Maple: two types of improper integrals as examples, Proceedings of 2013 Information Technology and Management Symposium, Taiwan, B9-B14, 2013.

[15] C. -H. Yu, The evaluation of two types of multiple improper integrals, Proceedings of 2012 Chang Yun Ka Alliance of Colleges Symposium, Taiwan, M-7, 2012.

[16] C. -H. Yu, Application of Maple: the evaluation of double integral as an example, Proceedings of 2013 International Symposium on Intercultural Communication, Taiwan, pp. 294-302, 2013.

[17] C. -H. Yu, Application of Maple: double improper integrals as examples, Proceedings of 2013 Information Education and Technology Application Conference, Taiwan, pp. 1-5, 2013.

[18] C. -H. Yu, Application of Maple: multiple integrals as examples, Proceedings of the 6th Cross-Strait Technology and Humanities Education and University-Industry Cooperation Seminar, Taiwan, EMD001, 2012.

[19] C. -H. Yu, Application of Maple on solving some type of multiple integrals, Proceedings of the 8th Information Management and Practice Conference, Taiwan, TP20120128, 2012.

[20] C. -H. Yu, Application of Maple on evaluating the double improper integrals, Proceedings of the Innovative Education and Learning Technology, 2013.

[21] C. -H. Yu, Application of Maple on evaluating multiple improper integrals, Proceedings of the 6th IEEE/International Conference on Advanced Infocomm Technology, Taiwan, No. $00282,2013$. 
[22] C. - H. Yu, Application of Maple on the evaluation of four types of double integrals, Proceedings of 2013 Business Innovation and Development Conference, Taiwan, B20130117002, 2013.

[23] C. -H. Yu, Using Maple to study multiple improper integrals, International Journal of Research in Information Technology,
Vol. 1, Issue. 8, pp. 10-14, 2013.

[24] T. M. Apostol, Mathematical Analysis, 2nd ed., Boston: Addison-Wesley, p269, 1975. 